\title{
An Integrative Review of Contemporary Perspectives on Videoconference-Based Therapy—Prioritising Indigenous and Ethnic Minority Populations in the Global South
}

\author{
Ben Classen $^{1}\left[\right.$ [ $\cdot$ Keith Tudor $^{1} \cdot$ Elizabeth du Preez ${ }^{1} \cdot$ Elizabeth Day $^{1} \cdot$ Julia loane $^{1} \cdot$ Brian Rodgers $^{1}$
}

Received: 9 December 2020 / Revised: 14 March 2021 / Accepted: 15 April 2021 / Published online: 21 April 2021

(c) The Author(s), under exclusive licence to Springer Nature Switzerland AG 2021

\begin{abstract}
An integrative literature review was undertaken as a means of drawing together contemporary perspectives on the outcomes and affordances of videoconference-based therapy. This review was conducted in a way which placed emphasis on the need for mental healthcare strategies which are mindful of the cultural and social needs of indigenous and ethnic minority populations, particularly those situated in the Global South. The review was undertaken using an inverse funnelling approach which sought to prioritise literature on videoconference-based therapy literature which specifically focused on indigenous and ethnic minority populations. A series of general and population specific searches across relevant health databases were supplemented by a simultaneous search of Google Scholar. The PICOS search tool was used in developing the search terms, and data was processed using an inductive approach to thematic analysis. A final dataset of 43 articles were included in the review. This body of literature encompassed an international range of studies and included perspectives informed by quantitative, qualitative and mixed methods research. Four key themes were identified across the reviewed literature: indigenous and ethnic minority populations, therapeutic relationships, clinical outcomes and technical and logistical considerations. Based on our findings, there is reason to believe that videoconference-based therapy can be made to be just as effective as offline, face-to-face modes of delivery. However, research into the efficacy, impact and cultural implications of this technology in relation to indigenous and ethnic minority populations represents a significant gap within contemporary literature.
\end{abstract}

Keywords Videoconference therapy $\cdot$ Online therapy $\cdot$ E-mental health $\cdot$ Ethnic minority $\cdot$ Global South

The exponential spread of high-speed Internet technology in recent decades has opened up new opportunities for providing healthcare services at a distance (Ignatowicz et al., 2019). The delivery and optimisation of telehealth services have received considerable attention across a range of health-related disciplines (Wosik et al., 2020), the value of which has become increasingly salient in the wake of the coronavirus (COVID-19) pandemic. In the era of alert levels, "lockdowns", restrictions on travel and social distancing requirements, delivering effective and efficient care via the Internet has quickly shifted from a marginal to a necessary capability, hastening the need for evidence-based strategies and policy for online care.

Ben Classen

ben.classen@vuw.ac.nz

1 Auckland University of Technology, Auckland, New Zealand
Of particular interest to mental health practitioners has been the availability of videoconference technology (Berryhill et al., 2019a, b). With the ability to provide real-time audio and visual communication between client and therapist, videoconference software allows for reciprocal communication similar to that experienced in traditional therapeutic settings (Lozano et al., 2015). Promising in terms of convenience and accessibility, the use of telehealth in the provision of mental health care has been identified by a number of governmental agencies as a means of expanding treatment options (Department of Health \& Ageing, 2012; Te Pou, 2018; Wozney et al., 2017). Despite this, prior to the COVID-19 pandemic, the provision of online care had often been relegated to a secondary option for care in professional mental health settings (Uscher-Pines et al., 2020). As clients and therapists alike have no option but to adapt to working online, there is renewed need for understanding contemporary perspectives on and outcomes of online therapy. This need is particularly pertinent in relation to persistent gaps 
in the literature surrounding clinical outcomes for traditionally underserved populations throughout the world.

The present article reports on an integrative review of recent research into online therapy. Given our research team's location in Aotearoa New Zealand, we recognised a need for a review of the literature that was mindful of the needs and nuances of mental health practice in the Global South. Although there already exists some insight into the nature of online therapy practice here in Aotearoa New Zealand (Babbage et al., 2020; Samuels, 1999), a significant majority of literature in this area focuses on outcomes and experiences across globally disparate contexts (Christensen et al., 2020; Connolly et al., 2020). As is the case around the world (Watson et al., 2019), indigenous and ethnic minority populations in the Global South are consistently overrepresented in negative mental health statistics, an enduring consequence of systemic inequality (Williams et al., 2018). Prioritising effective and culturally appropriate ways of improving outcomes for these populations, whilst also striving to expand and strengthen mental health strategies for all, is fundamental to Aotearoa New Zealand's mental health sector (Paterson et al., 2018). This endeavour has parallels with similar concerns in other countries, and thus, we believe this research has something to offer beyond the Global South.

\section{Definitions}

Throughout the literature, videoconference-based therapy is discussed according to a broad range of terms including, but not limited to teletherapy (Ghosh et al., 1997), e-mental health (McGinty et al., 2006), Internet therapy (King et al., 2009), telepsychiatry (Chan et al., 2015), telemental health (Connolly et al., 2020) and telepsychotherapy (Watts et al., 2020). In many cases, these terms are used to refer to not only videoconference-based care, but also the use of app-based (Kenny et al., 2015), telephone-based (Langarizadeh et al., 2017) and SMS or text-based care (Ganapathy et al., 2020). This has led to what has been referred to as a state of terminology chaos within the field (Smoktunowicz et al., 2020), as different approaches to, and definitions of, online care overlap with one another. For the purposes of the present review, we were primarily interested in videoconference-based forms of online therapy, wherein a client and therapist use software to interact with one another via synchronous audio and visual communication.

\section{Methodology}

This review is informed by a recognition of the value of theoretical perspectives which are mindful of the needs and nuances of mental healthcare within the Global South. In this, the authors are variously informed by critical theory and its application to research (Morrow \& Brown, 1994), and, given our geographical location, specifically Southern theory (Connell, 2007) and post-colonial theory and its underpinning of indigenous methodologies (Denzin et al., 2008; Smith, 2012). These perspectives are consistent with the social model of health (World Health Organisation, 1986, 2010) as a framework that accounts for cultural, political, economic and related determinants of health, and goals for sustainable development (Stafford-Smith et al., 2017) that aim to reduce inequity in the service of global wellbeing. However, both Southern and indigenous theories and methodologies offer a critical perspective on the supposed neutrality and centrality of the production of knowledge from the Western intellectual tradition and, in effect, the Global West and North (Akena, 2012). In order to address this - and redress this implicit bias in the literature-we adopted an inverse funnelling approach for our review with broader criteria to capture more local and nearby research (in Aotearoa New Zealand and Australia) on indigenous and ethnic minority research before tightening the criteria as we extended this out to the Global South and then the Global North.

As our intention in designing the review was to keep the experiences of minority populations at the forefront of our inquiry, the authors also recognise the inherent limitations associated with using published literature as a means of reviewing indigenous and ethnic minority perspectives. Our intention in designing these methods was that our findings might provide a foundation for future dialogue and engagement with ethnic and indigenous populations with a vested interest in mental health outcomes.

With regard to the subject of our enquiry, after preliminary reading and discussion, the authors discerned a need for a review of relevant literature which accounted for both the quantitative and qualitative aspects of therapy delivered via videoconference. Prior reviews of online therapy literature have primarily focused on empirical measures of outcomes and efficacy (Berryhill, 2019a, b; Te, 2018). Although such approaches provide valuable insight into readily quantifiable aspects of online care, in many cases, they do not appear to provide a holistic account of client and therapist experiences of online therapy. Given this, an integrative review was chosen as the most appropriate methodological approach. Widely used within the health sciences (Hopia et al., 2016), rigorous integrative reviews provide a means of drawing together evidence from a range of methodologically diverse research, with the intention of establishing an evidence-based perspective which goes beyond a review of strictly quantitative literature (Whittemore \& Knafl, 2005). In the interest of replicability and transparency, every effort was made to keep our review methods as systematic as possible. 


\section{Method}

\section{Search Strategy}

The PICOS search tool was chosen as a means of providing a clearly defined and empirically validated framework for the review's search strategy (Methley et al., 2014). The PICOS tool comprises five categories for structuring systematic searches (population, intervention, comparison, outcome and study type). The focus of the review was first aligned with each of these categories, and a search strategy was created which included a range of terms relevant to each of the categories. A full account of the terms used can be found in Appendix 1. This search strategy was then applied to four health science-related databases (EBSCOhost, PsycInfo, PubMed and Web of Science), the data from which formed the initial search results.

\section{Population-Specific Searches}

In order to enact the inverted funnel approach to counter the predominance of literature from the Global North, the PICOS search strategy was modified to include additional population-specific searches. In addition to these modified search criteria, additional sources such as books and relevant grey literature were searched for material not typically accessible through traditional commercial or academic publishing and distribution channels. The first of these searches started by focusing locally on Māori ${ }^{1}$ populations within Aotearoa New Zealand, the second was expanded slightly to focus on Pasifika/Pacific Islander ${ }^{2}$ populations and the third population-specific search had a broader focus on online therapy outcomes and experiences for a diverse range of indigenous and ethnic minority populations.

These population-specific searches used fewer keywords relating to the latter categories of the PICOS tool (i.e. comparison, outcome and study type). By keeping the focus of these searches on online therapy, while broadening our criteria regarding the type of literature being searched, we improved our chances of finding literature generally relevant to our study aims, and specifically relevant to ethnic minority populations. A complete list of the population search strategies can be found in Appendix 1.

\footnotetext{
${ }^{1}$ Indigenous people of Aotearoa New Zealand.

2 The Pasifika/Pacific Island populations includes those who are either migrants or descendants from the sub-regions of OceaniaPolynesia, Micronesia and Melanesia.
}

\section{Google Scholar}

After completing the general and population-specific searches, we also ran our search terms through Google Scholar's online search tool. Historically, concerns regarding search reliability (Neuhaus \& Daniel, 2008) and a lack of transparency (Jacsó, 2005) have caused Google Scholar to be overlooked as a serious, stand-alone option for conducting literature reviews (Bar-Ilan, 2019). Despite these concerns, recent research has pointed to the reliability of Google Scholar searches being comparable to traditional database searches (Harzing, 2013; Martín-Martín et al., 2018). Google Scholar has also been shown to increase the likelihood of retrieving grey literature relevant to a specific research enquiry (Shultz, 2007). Given this, we opted also to use Google Scholar as a means of supplementing our existing database search strategies. Due to Google's character limit on searches, and without the option to conduct complex multi-field searches, our Google Scholar search comprised fewer search terms. However, all Google Scholar terms were consistent with our initial database search strategy. A full account of these terms and our process for pre-screening Scholar results can be found in Appendix 1.

\section{Exclusion Criteria}

Exclusion criteria were primarily informed by our originally identified PICOS search terms; any articles which were not consistent with at least four of the five PICOS categories were excluded from the dataset. In order to keep the focus of the review on discourse surrounding contemporary perspectives and approaches to videoconference-based therapy, articles published prior to 2015 were excluded. Additional exclusion criteria included the removal of results which did not primarily focus on video conference-based forms of online therapy (e.g. asynchronous consultations and appbased interventions), as well as results which focused on blended or multi-modal forms of online therapy (e.g. a mixture of videoconference and face-to-face sessions).

\section{Analysis}

An inductive approach to thematic analysis (Braun \& Clarke, 2006) was identified as the most appropriate means for analysing the literature gathered from the database searches. An inductive approach to conducting the thematic analysis entailed allowing the initial coding and theme development process to be driven by the content of the gathered literature. Using this approach was deemed appropriate as it allowed for openended development of themes and findings, whilst reducing the likelihood of preconceived assumptions about the efficacy of online therapy outcomes colouring the analysis. Coding of the results was shared amongst the co-authors. Based on the final 
dataset and coding results, and in order to ensure an inductive approach to developing themes (Braun \& Clarke, 2006), individual co-authors made recommendations regarding emergent themes. These individual recommendations were then drawn together and grouped into consistently identified and significant themes. Only minor disparities were found between coauthors' recommendations regarding dominant themes, and these differences were resolved by group consensus regarding broadening the scope of more dominant themes to ensure they also accounted for less prescient trends within the data.

\section{Results}

The combined results of the general, population-specific and Google Scholar searches totalled 1253. Metadata for all results were imported into a reference management software (Zotero). A total of 290 duplicate results were identified and removed prior to the screening process. Screening of the remaining 963 articles was undertaken by the primary author, applying exclusion criteria whilst analysing the content of titles and abstracts. After excluding 793 results through this screening process, the remaining 170 articles were coded in Excel. Through this process, an additional 127 articles were identified and excluded according to our exclusion criteria, leaving a final dataset of 43 (Fig. 1). Analysis of this dataset by the research team resulted in 15 coding categories. Recurring conceptual themes within these categories were then discussed amongst the team resulting in the following four key themes: minority population outcomes, therapeutic relationships, clinical outcomes and technical and logistical considerations.

\section{Overview of Final Dataset}

The final results included a methodologically diverse dataset comprising 12 quantitative studies, six qualitative studies, eight mixed methods, 15 review articles and two book chapters. Despite our intentional search strategy, we located only one article from Aotearoa New Zealand (Babbage et al., 2020) and two articles from Australia (Dham et al., 2018; Simpson et al., 2015). This reflects the limited degree of contemporary evidence-based discourse in the context of the Global South, whilst the significant majority of articles reported on research conducted in the Global North. Articles tended to focus on the implications of videoconference therapy for either mental healthcare consumers $(n=21)$ or providers $(n=15)$, although some articles drew upon the experiences and outcomes of both parties $(n=10)$. Studies looked at the use of videoconference therapy across a wide range of mental health issues including depression (Christensen et al., 2020), anxiety (Berryhill et al., 2019a, b), OCD (Matsumoto et al., 2020) and post-traumatic stress disorder (PTSD) (Morland et al., 2015). An assortment of therapeutic interventions was also covered by the reviewed literature including cognitive processing therapy (Glassman et al., 2019), prolonged exposure therapy (Wells et al., 2020), general psychotherapy (Bouchard et al., 2020), psychiatry (Mahmoud et al., 2020) and group therapy (Heermann et al., 2016).

Though our search methods prioritised indigenous and ethnic minority populations, our final dataset included a very limited number of articles to draw from. Only four articles provided any significant insight into, or consideration of, the use of videoconference-based therapy amongst indigenous and ethnic minority populations, and of these, only one was focused specifically on ethnic minority outcomes (Dawson et al., 2020). This finding is consistent with the authors' initial impressions of a relative lack of evidence in this area of online therapy. Other domains of knowledge covered in the broader literature included the effectiveness of online therapeutic relationships, the clinical outcomes associated with providing online care and the logistical implications of online care.

Fig. 1 Summary of search results

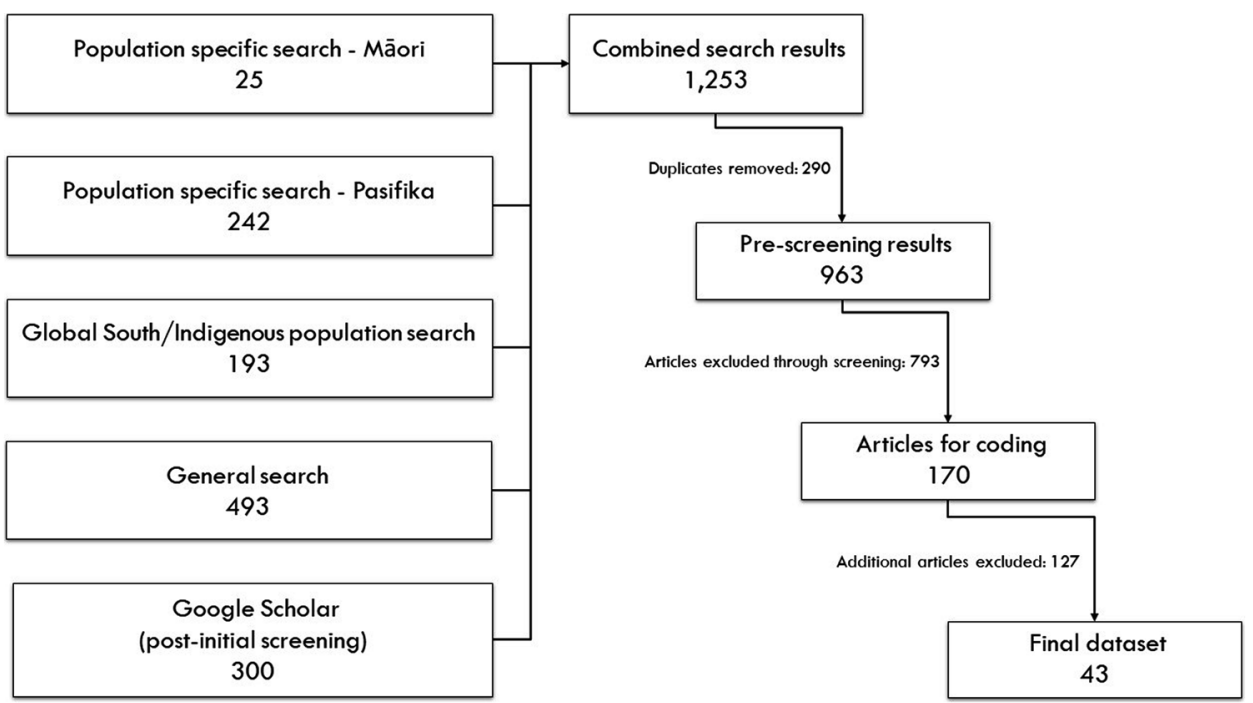




\section{Theme-Indigenous and Ethnic Minority Populations}

Our findings indicated a dearth of contemporary literature on online therapy outcomes for indigenous and ethnic minority populations within the Global South. Results did, however, include some insight into online therapy outcomes across international ethnic minority populations. One of the most attractive features of online therapy would appear to be its potential to provide care for underserved populations, and within our dataset, there was general recognition of the need for more research into which specific populations might be best served by the provision of online care (Kocsis \& Yellowlees, 2018). Although our searches identified a limited amount of recent work surrounding ethnic minority outcomes, research relating to several ethnic American minority groups was included in Chan et al. (2015) review of literature published between 2011 and 2015. Although focused on minority populations within America, this review highlighted the value of approaches to online therapy which are mindful of culturally diverse perspectives and experiences in a global context. Chan et al. (2015) noted that indigenous American populations in particular have benefitted from telepsychiatry programmes which have been based on ongoing collaboration with tribal elders, suggesting the potential for similar approaches to online therapy amongst minority groups here in the Global South.

A more recent review of indigenous telehealth by Dawson et al. (2020) covered literature which focused on the outcomes of clinical trials for both mental and physical health. In addition to the inclusion of physical health, a broad definition of telehealth was used which included telephone and SMS-based care. Despite these broad search criteria, only 6 relevant articles were identified by Dawson et al. most of which were based on physical, rather than mental, health outcomes. Although there are notable methodological discrepancies between Dawson et al.'s work and the present study, findings from both reviews point to the considerable lack of rigorous insight into long-term telehealth outcomes for indigenous and ethnic minority populations. Dawson et al. point to the significant potential for online therapy to provide cost-effective and accessible care to a wide range of ethnic minorities, including those living in remote areas.

Within our results, a small number of studies based on ethnically diverse samples did provide some degree of direct insight into outcomes for minority groups. However, few of these were focused specifically on outcomes for ethnic minorities, and findings were based on only minimally ethnically diverse samples (Heermann et al., 2016; Morland et al., 2015). Stewart et al. (2017) study of videoconference cognitive behavioural therapy (CBT) was one exception to this. This pilot study focused primarily on client satisfaction and treatment outcomes for underserved and underrepresented populations. Across a small sample of adolescents, consisting of 46\% Hispanic and 40\% African American participants, online therapy was demonstrated to be an effective means for improving PTSD symptom severity amongst ethnically diverse populations. Attempts to tailor the online therapy programme to best suit the cultural perspectives of participants were also evident in this study. This included the use of online therapy sessions as a means of addressing participant concerns surrounding ethnocultural beliefs and attitudes towards mental health treatment. Such initial results hold promise for the general feasibility of online therapy amongst minority populations, whilst also highlighting the need for future work into effective approaches to tailoring online care to best suit the needs of these underserved groups.

\section{Theme-Therapeutic Relationships}

The potential for videoconference-based therapy to impact the quality of the client/therapist relationship was one of the most widely reported concerns throughout the reviewed literature (Hoffmann et al., 2020; Kocsis \& Yellowlees, 2018; Mahmoud et al., 2020). These concerns were shared by both clients (Mahmoud et al., 2020) and therapists (McClellan et al., 2020), although the extent to which each of these parties were concerned about online therapy's impact on the working relationship, and the reason for this concern appeared to vary between the two groups. In a study comparing client and professional perceptions of telemental health services, Petersen et al. (2020) found that clients were slightly more comfortable with the idea of using videoconferencing than therapists. Although few studies simultaneously compared feedback from clients and therapists in this way, this pattern of mental health professionals expressing slightly more trepidation regarding effective online relationships appeared to be a trend reflected across the reviewed literature. Concerns regarding difficulty building a working relationship were more consistently associated with therapist perspectives (Goldstein \& Glueck, 2016; Kocsis \& Yellowlees, 2018; McClellan et al., 2020), and, although clients did express similar concerns (Mahmoud et al., 2020), they appeared to be more willing to engage with videoconference modes of delivery (Rozental et al., 2015).

A variety of reasons for doubting the quality of online relationships were identified throughout the literature. In a study of practitioners with experience providing care via videoconference, Feijt et al. (2020) identified a lack of non-verbal cues as one of the most widely reported issues with online therapy. The ability to read non-verbal signals including posture, body language, demeanour, and even smell are essential features of a therapist's toolkit, and these cues can be difficult and some impossible to gauge via videoconference. Concerns regarding difficulty reading non-verbal cues with regard to relationship building were 
consistently reported across studies of therapist perspectives (Békés et al., 2020; Feijt et al., 2020; Hoffmann et al., 2020), but appeared less often in studies of client perspectives (Mahmoud et al., 2020; Théberge-Lapointe et al., 2015). Interestingly, this may provide some explanation regarding the slight asymmetry between client and therapist scepticism of online therapy. The need to interpret and respond to nonverbal cues may be of less imminent concern to clients, making them more open to the idea of developing a therapeutic relationship via telepresence. Besides non-verbal cues, concerns regarding other aspects of the therapeutic relationship were evident and included anticipated issues with communicating empathy (Bouchard et al., 2020), expressing warmth (Lozano et al., 2015) and impersonality of care (Connolly et al., 2020).

Despite articles highlighting the challenges associated with online therapeutic relationships, a number of studies reported comparable working alliance outcomes across online and face-to-face contexts (Bouchard et al., 2020; Murphy \& Turgoose, 2019; Stewart et al., 2017; Watts et al., 2020). This contrast within the literature appeared to be at least in part due to a number of studies reporting upon the perspectives of therapists with no discernible first-hand experience in providing care via videoconference (Hoffmann et al., 2020; Maurya et al., 2020). Although there is value in understanding these concerns, in terms of implementing effective online care, it is important that the concerns and perspectives of therapists pre- and post-experience with online therapy are not conflated.

The literature suggests that initial scepticism towards capacity for relationship building tends to diminish after therapists have had a chance to engage with clients via videoconference (Lozano et al., 2015). Békés et al. (2020) provided a recent example of the value of assessing therapist perspectives on the therapeutic relationship post-experience with online care. A total of 190 mental health professionals who had moved to using videoconferencing due to the COVID-19 pandemic were asked to compare their experiences pre- and post-transition to online care. A majority of participants reported feeling as connected and authentic in their online sessions compared to pre-COVID face to face sessions. As mental health practice is forced to adapt to the era of social distancing, such work reflects the value of research in this area which prioritises practical and real-life experience over theoretical scepticism and assumptions.

\section{Theme-Clinical Outcomes}

Closely related to effective online relationships were the clinical outcomes associated with providing online care. This theme broadly encompassed client wellbeing, satisfaction, quality of life and symptom severity post-treatment. Broadly, client wellbeing refers to a client's emotional response to their concerns, while quality of life refers to the cognitive appraisal someone makes about the impact their health has on their everyday life, for further discussion of which see (Upton \& Upton, 2015). Comparing these clinical outcomes across online and face-to-face contexts was a consistent focus throughout the reviewed literature. Overall, clinical outcomes post-online therapy tended to be at least comparable with face-to-face therapy outcomes. A variety of methodological approaches were used to evaluate clinical outcomes ranging from randomised control trials (Morland et al., 2015), through to longitudinal case studies (Mahmoud et al., 2020). Clinical outcomes were assessed using a range of measures including established symptom severity measures (Matsumoto et al., 2020) and feedback from client families and caregivers (Mahmoud et al., 2020).

As one of the most readily quantifiable of clinical outcomes, symptom severity post-online treatment was frequently assessed throughout the literature. Reduced symptom severity was demonstrated across online interventions for PTSD (Chan et al., 2015; Morland et al., 2015; Murphy \& Turgoose, 2019), depression (Aly, 2017; Bouchard et al., 2020; Stewart et al., 2017) and OCD (Aly, 2017; Matsumoto et al., 2020), and these reductions were consistently found to be comparable with face-to-face outcomes. These findings point to the clinical efficacy of videoconferencing as a means of delivering a variety of therapeutic interventions. Notably, some mental health challenges tend to lend themselves to online therapy (Wangelin et al., 2016). A salient example is the treatment of clients with anxiety and panic-related challenges such as agoraphobia. The ability to meet with a therapist from the comfort of home provides a promising avenue to care for such clients, and evidence suggests that these online approaches can help reduce symptom severity.

In a quantitative non-inferiority trial, Bouchard et al. (2020) evaluated the outcomes and treatment processes of online therapy for treating agoraphobia and generalised panic disorders. Participants in both online and in-person conditions received 60-min CBT sessions once a week for 12 weeks. Videoconference outcomes were found to be statistically consistent with face-to-face delivery across a battery of symptom severity measures including the Panic and Agoraphobia Scale (PAS), Mobility Inventory When Alone (MI) and the Body Sensations Questionnaire (BSQ), and these clinical improvements remained steady at 12-month follow-up. Similar comparisons between online and face-to-face symptom severity were also evident across a number of studies focused on social anxiety and anxietyrelated disorders (Berryhill et al., 2019a, b; Matsumoto et al., 2020; Stewart et al., 2017; Théberge-Lapointe et al., 2015; Wangelin et al., 2016; Watts et al., 2020).

Beyond symptom severity, several other clinical outcomes were identified. Although within the literature, these alternative means for assessing clinical outcomes tend to be 
overshadowed by symptom severity measures, awareness of the need for nuanced and dynamic perspectives on clinical outcomes was evident (Gros et al., 2018). One such example was found in studies which assessed client satisfaction with online therapy. Client satisfaction with online interventions was broadly comparable with face-to-face therapy treatment satisfaction (Lozano et al., 2015; Wangelin et al., 2016) and was not limited to consumers with pre-existing familiarity with videoconference technology. Recent work by Christensen et al. (2020) reviewed literature relating to client satisfaction amongst elderly clients suffering from depression. Despite being a demographic typically associated with low technological literacy, the review found overall high levels of satisfaction amongst elderly users of videoconference-based care. As with therapists' pre-treatment scepticism, concerns about online therapy outcomes appear to quickly dissipate amongst elderly clients after gaining first-hand experience with using the technology.

Throughout the literature surrounding clinical outcomes, there was also some consideration of online treatment adherence and attrition. In a recent survey of Dutch mental health providers with experience providing online care, several participants reported higher levels of adherence to treatment amongst online clients (Feijt et al., 2020). In relation to this finding, Feijt et al. also refer to the fact that online therapy enables the possibility of more frequent, brief contacts between client and therapist. While such regular and convenient contact may increase a client's likelihood of remaining engaged with, and adhering to a treatment plan, the reviewed literature lacked any concrete evidence supporting this implied causal link. Closely related to treatment adherence in online care were concerns regarding attrition rates amongst clients. Although there was some indication that rates of attrition were no more problematic amongst online clients (Wangelin et al., 2016), within our review, we found limited empirical data which explicitly supported this case. A pilot study by Stewart et al. (2017) was one exception to this, in which there was a $0 \%$ drop out rate amongst a small sample of 15 adolescents who received 12-20 weekly sessions of trauma-focused CBT via videoconference. Although such findings are promising, understanding the extent of attrition and adherence in online therapy treatment would benefit from more longitudinal and broadly generalisable insight.

Quality of life was a clinical outcome which, although identified as an important concern, was only reported upon sparingly throughout the literature, often featuring as a supplementary consideration alongside things like symptom severity (Banbury et al., 2018). This dearth of evidence on quality of life post-online therapy was noted in a study by Glassman et al. (2019) which assessed quality of life across male and female veterans with PTSD. Two separate randomised controlled trials (one consisting of 125 males and one of 126 females) compared quality of life scores for participants receiving online cognitive processing therapy compared with a control group which received the same treatment plan in a face-to-face setting. Across online and face-to-face therapy conditions, improvements in quality of life scores were comparable through to 6-month followup, indicating no significant difference in online therapy's capacity to improve quality of life outcomes. As with other clinical outcomes outside of symptom severity, the literature is generally optimistic about online therapy's capacity to improve quality of life (Banbury et al., 2018; Hilty et al., 2016). However, there is still considerable room for further insight into quality of life outcomes across specific populations and types of interventions.

Despite generally optimistic results across the literature, some negative aspects of clinical outcomes were identified. In a recent study of outpatient psychiatrists with first-hand experience of providing distanced care, a number of drawbacks to online care were identified (Uscher-Pines et al., 2020). While many of these limitations related to logistical and ethical implications of online care, there was evidence of some negative impact on clinical outcomes. Participants noted the potential for distanced care to obscure accurate and holistic assessments of client wellbeing, an issue which was noted as particularly problematic when prescribing medication and conducting appropriate assessment of newer clients. Although the data in this study was collected from a relatively small sample of participants, such findings point to the need for approaches to online therapy which are mindful of specific theoretical orientations to mental health care. Difficulty accurately prescribing appropriate medication in online therapy is likely to be of less concern to cognitive behavioural therapists than it is to psychiatrists. Similarly, concerns with client's ability to conduct self-guided gradual exposure tasks assigned via online CBT treatment-as reported by patients in a study by Rozental et al. (2015)—is likely to be of less concern to more prescription oriented psychiatrists. Contrasting clinical outcomes across different theoretical approaches to online care demonstrate that although broadly efficacious, online therapy should not be taken as a one size fits all approach to therapy.

\section{Theme-Logistical Considerations}

The logistical implications of online care were widely discussed throughout the literature, and among these, the potential for increasing service accessibility was a consistently identified benefit of providing online therapy (Dawson et al., 2020; Dham et al., 2018; Duffy, 2016). The widespread use of smartphone and broadband/fibre optic technology means that more and more individuals have the means to engage with videoconference software (Wangelin et al., 2016). Accessibility of care was particularly relevant in 
studies which focused on clients living in isolated areas. In a study of clients living in rural and remote areas (Dham et al., 2018), high patient satisfaction with online therapy was reported, and accessibility was identified as one of the most significant benefits for socially isolated individuals. Similarly, a survey of rural New Zealanders identified strong support for distanced mental healthcare amongst rural communities (Babbage et al., 2020). In contrast to these optimistic client perspectives, a study of attitudes towards telehealth care amongst rural mental health professionals identified a consistent degree of scepticism regarding the efficacy of telehealth care (McClellan et al., 2020). McClellan et al. note that although telehealth has the potential to drastically improve accessibility to care in rural areas, clinician adoption and attitudes are a key determinant in the success of online therapies.

The importance of therapist competence in providing online therapy was a logistical concern which was echoed across the literature. Relevant professional training is significantly correlated with positive views towards online therapy (Békés et al., 2020), suggesting that as professional support and development continues to improve, so too will therapists' attitudes and engagement. In a qualitative study of learning needs amongst postgraduate psychiatry residents, Crawford et al. (2016) identified a number of important aspects of telepsychiatric training including technical, assessment, relational and communication skills in online therapeutic environments. While the development of postgraduate training towards the provision of online care is likely a step in the right direction, ensuring there is sufficient support for currently practicing therapists is vital for the uptake and advancement of online therapies (Hoffmann et al., 2020). Issues with accessing training and resources were identified as key barriers to the uptake of online therapy in a review of telepsychiatry outcomes (Hubley et al., 2016), and similar concerns were consistently identified across studies reporting on therapist perspectives on online care (Alicata et al., 2016; Connolly et al., 2020; Hoffmann et al., 2020; Maurya et al., 2020).

The nature of online therapy gives rise to several unique logistical considerations, not least among which is the potential for technical issues associated with video and audio quality. Unreliable network connections have the potential to impede the efficacy of online therapy (Watts et al., 2020), and problems associated with poor video and audio quality were a noteworthy concern amongst both clients (Dham et al., 2018) and therapists (Uscher-Pines et al., 2020). These concerns highlight the importance of videoconferencing equipment and software which is reliable and user friendly (Bouchard et al., 2020). The requisite purchase, setting up and training of clinicians to use this software and technology in turn raises questions surrounding the initial costs associated with implementing online care (Berryhill et al., 2019a, b). Despite the cost of up-front investment, long-term online therapy was widely regarded as a cost-effective form of care (Alicata et al., 2016; Dawson et al., 2020; Matsumoto et al., 2020; Maurya et al., 2020), due to a reduced need for resources such as office space and administrative support (Hubley et al., 2016). There is also potential for this costeffectiveness to be passed on to consumers thanks to both reduced overheads for providers, and in the form of reduced travel costs for clients (Hilty et al., 2016). However, evidence of the extent to which these savings are passed on to clients was lacking within the reviewed literature.

Another logistical consideration which featured throughout the literature was the nature of privacy in providing online therapy. Videoconference-based therapy allows clients and therapists to connect from the comfort of their homes, potentially providing a level of personal insight which is often not as readily available in traditional therapeutic relationships. This window into a client's private life was associated with both positive and negative implications for practice. In one study, therapists reported that some clients appeared to be more comfortable with self-disclosure in their own homes (Feijt et al., 2020). Additionally, the opportunity to see how a client behaves and interacts with their home environment has the potential to provide nuanced insight into their character which might not be otherwise obvious in a clinical setting (Feijt et al., 2020). Conversely, some clients may feel as though their privacy is being invaded by a therapist (Christensen et al., 2020). In many cases, the home environment of a client may not be conducive with a private therapeutic discussion due to distractions and interruptions which wouldn't be otherwise present in a clinical setting (Uscher-Pines et al., 2020). These logistical considerations are further complicated in the context of online group therapy, wherein issues with the background environments of a client may be multiplied by the number of individuals in a group therapy session (Weinberg, 2020). These findings highlight the need for professional practice which is conscious of and responsive to the environment in which a client engages with online therapy.

\section{Discussion}

The reviewed literature provides reason to be optimistic about the capacity of online therapy to provide clinical outcomes which are comparable with traditional, in-person therapy. Although most studies report general trepidation from both therapists and clients, these concerns appear to be best overcome with first-hand experience engaging with online therapy. Evidence supports the potential for developing productive therapeutic relationships, and clinical outcomes are consistently found to be comparable with face-toface therapy across outcome measures for a variety of types 
of treatment. Online therapy presents a number of unique logistical and technical issues, although none of the issues identified within the literature appear to be insurmountable given appropriate resource allocation and implementation strategies.

However, of significant concern is the lack of research specific to indigenous and ethnic minority communities. These populations continue to be disproportionately represented in mental health statistics both globally (Jankovic et al., 2020), and here in Aotearoa New Zealand (Parker, 2019). Addressing this disparity calls for the provision of mental health care which is both culturally sensitive (Naeem et al., 2019) and widely accessible (Mercer et al., 2019). As a rapidly developing and dynamic approach to providing care, online therapy has the potential to make a valuable contribution to tackling both of these systemic deficits. A key observation made by Dawson et al. (Dawson et al., 2020), in this regard, is the importance of critical perspectives on telehealth's capacity for accessible care. Although availability is a central tenet of accessible care, to be truly accessible, a service must also be culturally acceptable and appropriate. Furthermore, given the inequities that generally exist among indigenous and ethnic populations, it will be crucial to address the digital disparities that exist amongst such communities (Cullen, 2001). For example, in Aotearoa New Zealand, reports highlight the lack of digital resources among Māori and Pacific communities (Digital Inclusion Research Group, 2017). Without specific studies that engage with indigenous and ethnic minority populations, there is real danger that findings from research conducted in the Global North on the effectiveness of online therapeutic relationships, the clinical outcomes associated with providing online care, and the logistical implications of online care will be considered universal, and indiscriminately applied to populations in the Global South.

There is reason to believe that the ongoing COVID-19 pandemic will mark an important turning point in the provision of online mental health care (Wind et al., 2020). As clients are forced to accommodate continual shifts in social distancing requirements, proficiency and confidence in using videoconference technology is becoming an essential skill (Reay et al., 2020). Additionally, rapid regulatory changes in response to the pandemic mean that logistical and financial barriers to implementing online care are being quickly overcome (Haque, 2020). These changes bode well for the future of online therapy, as evidence suggests that as clients and therapists become more comfortable with videoconferencebased therapy, the efficacy and outcomes of such measures will improve.

Despite the general optimism evident across the findings of this review, it is important to note that much of this research reports on small sample sizes and in many cases limited longitudinal insight. Outside of symptom severity, clinical outcomes such as client satisfaction, adherence, attrition, and quality of life are vital aspects of online therapy. Understanding how these outcomes manifest and change over time and across broad population groups will likely be an important direction for future research. Additionally, findings which are only mentioned in passing within the reviewed literature- such as increased burnout amongst therapists (Békés et al., 2020)—will be important to explore further so as to ensure online therapy can be provided in a sustainable fashion. As the uptake and implementation of online therapy continues to spread post-pandemic, there is increased potential for niche concerns and methodological gaps within the literature to be addressed. With the largescale rollout of online therapy, future research surrounding long-term and population specific outcomes will become increasingly viable.

\section{Limitations of Present Study}

While the focus of this review was videoconference-based therapy, it is important to note that online mental health care includes a diverse range of modalities and approaches to care (Carl et al., 2019; Kenny et al., 2015; Possemato et al., 2011). Furthermore, many of these different approaches to online care include strategies with the potential to be specifically tailored to provide for underserved minority populations (Fleming et al., 2012; Povey et al., 2016). The present review was therefore limited in that it provided insight into just one facet of the broad corpus of literature on general online care. It is plausible that online care which does not solely consist of videoconference-based therapy (e.g. interventions in which videoconferencing is used alongside other strategies) may provide outcomes and practical challenges beyond those covered in the present review.

The data included in the final dataset was limited to articles published post-2015. This methodological decision was made in order to keep the focus on contemporary outcomes and perspectives with an attempt to prioritise indigenous and ethnic minority voices. Based on the reviewed literature, contemporary practice outcomes for online therapy appear to be largely consistent with findings published prior to 2015 , and it seems unlikely that the review's key findings would have been drastically different had our dataset drawn on earlier work within the field. Despite this, accounting for perspectives and outcomes published pre-2015 may prove valuable in terms of future efforts to develop evidence-based practice and policy. Additionally, the present study both benefitted and was limited by its proximity to the global COVID-19 pandemic. The recent rapid implementation of online therapy in response to social distancing requirements signals new horizons for online healthcare. Although the current review included some valuable insight into postpandemic online therapy, future research/reviews in this 
area will likely further benefit from the ongoing surge in experiences and outcomes relating to online care.

From the perspective of attempting to privilege indigenous and ethnic minority voices, a significant limitation of this review is the methodology itself. Literature reviews inherently rely on published research. Whilst seemingly obvious, publications are actually part of a complex process of knowledge production based in Western education systems which promote a global domination of what is considered "legitimate" knowledge (Akena, 2012). This influences what is published, where it is published, and how it is accessed. All these factors inherently bias the results of any search results. Despite our attempts to counter this with our search strategy, it is likely that significant domains of indigenous and ethnic minority knowledge around perspectives on videoconference-based therapy will be absent from this review. We hence recommend that further studies are conducted in consultation with indigenous and ethnic minority communities in the Global South using methodologies that privilege the voices of these communities. Examples of such approaches may involve extending insight into indigenous experiences and outcomes beyond those simply reported in formal academic publications. Doing so will likely involve seeking direct feedback from, and facilitating dialogue with, indigenous community representatives and focus groups.

\section{Concluding Statement}

Contemporary literature indicates that videoconferencebased therapy has the potential to provide mental healthcare which is effective, accessible and affordable. However, these findings are largely posited from the perspective the Global North and dominant Western discourses around efficacy and accessibility. Without further studies located in the Global South, and particularly with indigenous and ethnic minority communities such as Māori and Pasifika, any generalisations from the contemporary literature risk the perpetuation of colonising activity that privileges certain voices while silencing others.

\section{Appendix 1. Search Results and Exclusion Criteria}

\section{General Search}

("mental health" OR "mental wellness" OR "mental wellbeing" OR "mental illness") AND (consume* OR patient OR client OR practitione* OR therapis* OR professio*). (((internet OR online) AND (therapy OR psychotherapy OR counselling)) OR teletherapy OR telehealth OR e-therapy OR "e-mental health" OR videoconference OR "video conference" OR synchronous).

"face-to-face" OR "face to face" OR "in-person" OR "in person"

efficacy OR adherence OR outcomes OR experienc* OR "quality of care" OR "quality of life".

quantitative OR qualitative OR "mixed methods".

Final general results as@24/9/20:

EBSCOhost (all databases): 86.

PsycInfo (via ProQuest): 291.

Web of Science (all databases): 63.

PubMed: 53.

Total: 493.

\section{Population Specific Searches}

\section{Māori Populations}

"mental health" AND (māori OR maori).

(((internet OR online) AND (therapy OR psychotherapy OR counselling)) OR teletherapy OR telehealth OR e-therapy OR "e-mental health").

Final Maori results as @ 24/9/20:

EBSCOhost: 9

PsycInfo: 3

Web of Science: 4

PubMed: 9

Total: 25.

\section{Pasifika Populations}

"mental health" AND (pasifika OR pacific OR "pacific islan*" OR polynes*).

(((internet OR online) AND (therapy OR psychotherapy OR counselling)) OR teletherapy OR telehealth OR e-therapy OR "e-mental health").

Final Pasifika results as @ 24/9/20:

EBSCOhost: 60.

PsycInfo: 96.

Web of Science: 38 .

PubMed: 48.

Total: 242.

\section{Ethnic Minority Populations/Global South}

"mental health" AND (indigenous OR aborigi* OR "first nation" OR minori* OR ethni* OR "Global South" OR oceania OR australasia).

(((internet OR online) AND (therapy OR psychotherapy OR counselling)) OR teletherapy OR telehealth OR 
e-therapy OR "e-mental health" OR videoconference OR "video conference" OR synchronous).

"face-to-face" OR "face to face" OR "in-person" OR "in person"

Final ethnic/Global South results as @ 24/9/20:

EBSCOhost: 32.

PsycInfo: 34.

Web of Science: 46.

PubMed: 81.

Total: 193.

Total database search results for all searches: 953.

\section{Google Scholar Search}

"mental health" AND ((internet OR online) AND (therapy OR psychotherapy OR counselling)) OR teletherapy OR telehealth OR e-therapy OR "e-mental health") AND (videoconferen* OR "video conferen*" OR synchronous) -blended -game -text-based.

\section{Google Scholar Pre-screening}

The Scholar search resulted in $>6,000$ results. The titles and abstracts of these results were screened for relevance by one researcher in batches of 20. Relevance of articles was determined according to our exclusion criteria (detailed below). By the $15^{\text {th }}$ batch of Google Scholar results the number of relevant results per batch had dropped to $<15 \%$. This point was therefore determined to be an appropriate cut-off point, and metadata for the first 300 results of the Google Scholar search was extracted and added to the dataset.

Total Scholar results as @ 24/9/20: 6,970.

Exported first 300 results and added to full search results in Zotero.

Combined results from Scholar and database searches: 1253.

All results combined and de-duped in Zotero.

Duplicate results removed: 290.

All data pre-screening: 963 .

Articles removed through first round of screening: 793.

Articles removed through second round of screening (whilst coding): 127.

Final coded dataset: 43.

\section{Exclusion Criteria}

The following types of literature will be removed from the search results:

- Articles which are not explicitly focused on online mental health interventions (i.e. those which focused on online general health practice).
- Articles which were not primarily focused on video conference-based forms of online therapy (i.e. asynchronous consultations, app-based interventions, self-guided online resources, e-mail, text, instant messaging, internet support groups, etc.)

- Articles which focus on Blended therapy (i.e. treatment protocols which consist of a mixture of face-to-face sessions and engagement with self-guided online resources)

- Articles which are primarily focused on the legal, logistical and technical implications of online therapy.

- Articles which are primarily focused on online methods for conducting training/education of mental health practitioners.

- Research articles which do not report on online therapy outcomes, adherence, or efficacy.

- Articles published prior to 2015.

- Non-English articles.

\section{Declarations}

Ethical Standards All research reported upon in this manuscript was conducted in a manner consistent with relevant ethical and academic standards. Given the nature of the study, approval from a specific ethical approval board was not deemed necessary.

\section{References}

Akena, F. A. (2012). Critical analysis of the production of Western knowledge and its implications for Indigenous knowledge and decolonization. Journal of Black Studies, 43(6), 599-619.

Alicata, D., Schroepfer, A., Unten, T., Agoha, R., Helm, S., Fukuda, M., Ulrich, D., \& Michels, S. (2016). Telemental health training, team building, and workforce development in cultural context: The Hawaii experience. Journal of Child \& Adolescent Psychopharmacology, 26(3), 260-265. CINAHL Complete. https://doi. org/10.1089/cap.2015.0036

Aly, R. (2017). Remote cognitive behavior therapy for obsessivecompulsive disorder in Egypt: A randomized trial. European Psychiatry, 41(S1), S147-S147.

Babbage, D. R., van Kessel, K., Terraschke, A., Drown, J., \& Elder, H. (2020). Attitudes of rural communities towards the use of technology for health purposes in New Zealand: a focus group study. British Medical Journal Open, 10(6), e037892. https://doi.org/10. 1136/bmjopen-2020-037892

Banbury, A., Nancarrow, S., Dart, J., Gray, L., \& Parkinson, L. (2018). Telehealth interventions delivering home-based support group videoconferencing: Systematic review. Journal of Medical Internet Research, 20(2), e25. https://doi.org/10.2196/jmir.8090

Bar-Ilan, J. (2019). Data Collection from the Web for Informetric Purposes. In W. Glänzel, H. F. Moed, U. Schmoch, \& M. Thelwall (Eds.), Springer Handbook of Science and Technology Indicators (pp. 781-800). Springer International Publishing. https://doi.org/ 10.1007/978-3-030-02511-3_30 
Békés, V., Aafjes-van Doorn, K., Prout, T. A., \& Hoffman, L. (2020). Stretching the analytic frame: Analytic therapists' experiences with remote therapy during COVID-19. Journal of the American Psychoanalytic Association, 68(3), 437-446. APA PsycInfo®. https://doi.org/10.1177/0003065120939298

Berryhill, B., Culmer, N., Williams, N., Halli-Tierney, A., Betancourt, A., Roberts, H., \& King, M. (2019a). Videoconferencing psychotherapy and depression: a systematic review. Telemedicine and E-Health, 25(6), 435-446.

Berryhill, B., Halli-Tierney, A., Culmer, N., Williams, N., Betancourt, A., King, M., \& Ruggles, H. (2019b). Videoconferencing psychological therapy and anxiety: a systematic review. Family Practice, 36(1), 53-63.

Bouchard, S., Allard, M., Robillard, G., Dumoulin, S., Guitard, T., Loranger, C., Green-Demers, I., Marchand, A., Renaud, P., \& Cournoyer, L.-G. (2020). Videoconferencing psychotherapy for panic disorder and agoraphobia: Outcome and treatment processes from a non-randomized non-inferiority trial. Frontiers in Psychology, 11, 2164.

Braun, V., \& Clarke, V. (2006). Using thematic analysis in psychology. Qualitative Research in Psychology, 3(2), 77-101.

Carl, E., Stein, A. T., Levihn-Coon, A., Pogue, J. R., Rothbaum, B., Emmelkamp, P., Asmundson, G. J., Carlbring, P., \& Powers, M. B. (2019). Virtual reality exposure therapy for anxiety and related disorders: A meta-analysis of randomized controlled trials. Journal of Anxiety Disorders, 61, 27-36.

Chan, S., Parish, M., \& Yellowlees, P. (2015). Telepsychiatry today. Current Psychiatry Reports, 17(11), 89-89. CINAHL Complete. https://doi.org/10.1007/s11920-015-0630-9

Christensen, L. F., Moller, A. M., Hansen, J. P., Nielsen, C. T., \& Gildberg, F. A. (2020). Patients' and providers' experiences with video consultations used in the treatment of older patients with unipolar depression: A systematic review. Journal of Psychiatric and Mental Health Nursing, 27(3), 258-271. https://doi.org/10. $1111 /$ jpm.12574

Connell, R. (2007). Southern theory: Social science and the global dynamics of knowledge. Cambridge: Polity.

Connolly, S. L., Miller, C. J., Lindsay, J. A., \& Bauer, M. S. (2020). A systematic review of providers' attitudes toward telemental health via videoconferencing. Clinical Psychology: Science and Practice, 27(2), e12311. https://doi.org/10.1111/cpsp.12311

Crawford, A., Sunderji, N., López, J., \& Soklaridis, S. (2016). Defining competencies for the practice of telepsychiatry through an assessment of resident learning needs. BMC Medical Education, 16, 28. https:// doi.org/10.1186/s12909-016-0529-0

Cullen, R. (2001). Addressing the digital divide. Online Information Review, 25(5), 311-320. https://doi.org/10.1108/1468452011 0410517

Dawson, A. Z., Walker, R. J., Campbell, J. A., Davidson, T. M., \& Egede, L. E. (2020). Telehealth and indigenous populations around the world: a systematic review on current modalities for physical and mental health. Mhealth, 6 .

Denzin, N. K., Lincoln, Y. S., \& Smith, L. T. (Eds.). (2008). Handbook of critical and indigenous methodologies. Sage.

Department of Health and Ageing. (2012). E-mental health strategy for Australia. https://www1.health.gov.au/internet/publications/ publishing.nsf/Content/mental-pubs-e-emstrat-toc

Dham, P., Gupta, N., Alexander, J., Black, W., Rajji, T., \& Skinner, E. (2018). Community based telepsychiatry service for older adults residing in a rural and remote region- utilization pattern and satisfaction among stakeholders. BMC Psychiatry, 18(1), 316. https:// doi.org/10.1186/s12888-018-1896-3

Digital Inclusion Research Group. (2017). Digital New Zealanders: The Pulse of our Nation (p. 105) [Report to NZ Govt agencies]. https://www.mbie.govt.nz/dmsdocument/3228-digital-new-zeala nders-the-pulse-of-our-nation-pdf
Duffy, D. Q. (2016). Prospective Clients' Expectations about Videoconference Psychotherapy. Southern Illinois University at Edwardsville.

Feijt, M., de Kort, Y., Bongers, I., Bierbooms, J., Westerink, J., \& W, I. J. . (2020). Mental health care goes online: Practitioners' experiences of providing mental health care during the COVID19 pandemic. Cyberpsychology, Behavior and Social Networking. https://doi.org/10.1089/cyber.2020.0370

Fleming, T., Dixon, R., Frampton, C., \& Merry, S. (2012). A pragmatic randomized controlled trial of computerized CBT (SPARX) for symptoms of depression among adolescents excluded from mainstream education. Behavioural and Cognitive Psychotherapy, 40(5), 529-541. APA PsycInfo®. https://doi.org/10.1017/S1352 465811000695

Ganapathy, S., de Korne, D. F., Chong, N. K., \& Car, J. (2020). The role of text messaging and telehealth messaging apps. Pediatric Clinics, 67(4), 613-621.

Ghosh, G. J., McLaren, P. M., \& Watson, J. P. (1997). Evaluating the alliance in videolink teletherapy. Journal of Telemedicine and Telecare, 3(1_suppl), 33-35.

Glassman, L. H., Mackintosh, M. A., Talkovsky, A., Wells, S. Y., Walter, K. H., Wickramasinghe, I., \& Morland, L. A. (2019). Quality of life following treatment for PTSD: Comparison of videoconferencing and in-person modalities. Journal of Telemedicine \& Telecare, 25(2), 123-127. Academic Search Alumni Edition.

Goldstein, F., \& Glueck, D. (2016). Developing rapport and therapeutic alliance during telemental health sessions with children and adolescents. Journal of Child and Adolescent Psychopharmacology, 26(3), 204-211.

Gros, D. F., Lancaster, C. L., López, C. M., \& Acierno, R. (2018). Treatment satisfaction of home-based telehealth versus in-person delivery of prolonged exposure for combat-related PTSD in veterans. Journal of Telemedicine and Telecare, 24(1), 51-55. APA PsycInfo®. https://doi.org/10.1177/1357633X16671096

Haque, S. N. (2020). Telehealth Beyond COVID-19. Psychiatric Services, appi.ps.202000368. https://doi.org/10.1176/appi.ps.20200 0368

Harzing, A.-W. (2013). A preliminary test of Google Scholar as a source for citation data: A longitudinal study of Nobel prize winners. Scientometrics, 94(3), 1057-1075. https://doi.org/10.1007/ s11192-012-0777-7

Heermann, C., Absenger, W., \& Sarris, J. (2016). Videoconference mindbody group therapy in a public mental health setting: a pilot study. Journal of Technology in Behavioral Science, 1(1-4), 37-42.

Hilty, D. M., Yellowlees, P. M., Myers, K., Parish, M. B., \& Rabinowitz, T. (2016). The effectiveness of e-mental health: Evidence base, how to choose the model based on ease/cost/strength, and future areas of research. In D. Mucic \& D. M. Hilty (Eds.), e-Mental health (1764349369; 2016-00366-006; pp. 95-127, Chapter xiv, 310 Pages). Springer International Publishing, Cham; APA PsycInfoß. https://search.proquest.com/docview/1764349369?accou ntid $=14782$

Hoffmann, M., Wensing, M., Peters-Klimm, F., Szecsenyi, J., Hartmann, M., Friederich, H.-C., \& Haun, M. W. (2020). Perspectives of psychotherapists and psychiatrists on mental health care integration within primary care via video consultations: Qualitative preimplementation study. Journal of Medical Internet Research, 22(6), e17569.

Hopia, H., Latvala, E., \& Liimatainen, L. (2016). Reviewing the methodology of an integrative review. Scandinavian Journal of Caring Sciences, 30(4), 662-669. https://doi.org/10.1111/scs.12327

Hubley, S., Lynch, S. B., Schneck, C., Thomas, M., \& Shore, J. (2016). Review of key telepsychiatry outcomes. World Journal of Psychiatry, 6(2), 269.

Ignatowicz, A., Atherton, H., Bernstein, C. J., Bryce, C., Court, R., Sturt, J., \& Griffiths, F. (2019). Internet videoconferencing for 
patient-clinician consultations in long-term conditions: A review of reviews and applications in line with guidelines and recommendations. Digital Health, 5, 2055207619845831. https://doi. org/10.1177/2055207619845831

Jacsó, P. (2005). Google Scholar: the pros and the cons. Online Information Review, 29(2), 208-214. https://doi.org/10.1108/14684 520510598066

Jankovic, J., Parsons, J., Jovanović, N., Berrisford, G., Copello, A., Fazil, Q., \& Priebe, S. (2020). Differences in access and utilisation of mental health services in the perinatal period for women from ethnic minorities-a population-based study. BMC Medicine, 18(1), 245. https://doi.org/10.1186/s12916-020-01711-w

Kenny, R., Dooley, B., \& Fitzgerald, A. (2015). Feasibility of" CopeSmart": a telemental health app for adolescents. JMIR Mental Health, 2(3), e22.

King, V. L., Stoller, K. B., Kidorf, M., Kindbom, K., Hursh, S., Brady, T., \& Brooner, R. K. (2009). Assessing the effectiveness of an Internet-based videoconferencing platform for delivering intensified substance abuse counseling. Journal of Substance Abuse Treatment, 36(3), 331-338.

Kocsis, B. J., \& Yellowlees, P. (2018). Telepsychotherapy and the therapeutic relationship: Principles, advantages, and case examples. Telemedicine and E-Health, 24(5), 329-334.

Langarizadeh, M., Tabatabaei, M. S., Tavakol, K., Naghipour, M., Rostami, A., \& Moghbeli, F. (2017). Telemental health care, an effective alternative to conventional mental care: A systematic review. Acta Informatica Medica, 25(4), 240.

Lozano, B. E., Birks, A. H., Kloezeman, K., Cha, N., Morland, L. A., \& Tuerk, P. W. (2015). Therapeutic alliance in clinical videoconferencing: Optimizing the communication context. In P. W. Tuerk $\&$ P. Shore (Eds.), Clinical videoconferencing in telehealth: Program development and practice (1661988642; 2015-00654-010; pp. 221-251, Chapter xxiii, 259 Pages). Springer International Publishing, Cham; APA PsycInfo®. https://search.proquest.com/ docview/1661988642? accountid $=14782$

Mahmoud, H., Naal, H., \& Cerda, S. (2020). Planning and implementing telepsychiatry in a community mental health setting: A case study report. Community Mental Health Journal, 1-7.

Martín-Martín, A., Orduna-Malea, E., Thelwall, M., \& Delgado LópezCózar, E. (2018). Google Scholar, Web of Science, and Scopus: A systematic comparison of citations in 252 subject categories. Journal of Informetrics, 12(4), 1160-1177. https://doi.org/10. 1016/j.joi.2018.09.002

Matsumoto, K., Hamatani, S., Nagai, K., Sutoh, C., Nakagawa, A., \& Shimizu, E. (2020). Long-term effectiveness and cost-effectiveness of videoconference-delivered cognitive behavioral therapy for obsessive-compulsive disorder, panic disorder, and social anxiety disorder in Japan: One-year follow-up of a single-arm trial. JMIR Mental Health, 7(4), e17157.

Maurya, R. K., Bruce, M. A., \& Therthani, S. (2020). Counselors' perceptions of distance counseling: A national survey. Journal of Asia Pacific Counseling, 10(2), 1-22. Academic Search Alumni Edition.

McClellan, M. J., Florell, D., Palmer, J., \& Kidder, C. (2020). Clinician telehealth attitudes in a rural community mental health center setting. Journal of Rural Mental Health, 44(1), 62-73. APA PsycInfo®. https://doi.org/10.1037/rmh0000127

McGinty, K. L., Saeed, S. A., Simmons, S. C., \& Yildirim, Y. (2006). Telepsychiatry and e-mental health services: potential for improving access to mental health care. Psychiatric Quarterly, 77(4), $335-342$

Mercer, L., Evans, L. J., Turton, R., \& Beck, A. (2019). Psychological therapy in secondary mental health care: Access and outcomes by ethnic group. Journal of Racial and Ethnic Health Disparities, 6(2), 419-426. https://doi.org/10.1007/s40615-018-00539-8
Methley, A. M., Campbell, S., Chew-Graham, C., McNally, R., \& Cheraghi-Sohi, S. (2014). PICO, PICOS and SPIDER: a comparison study of specificity and sensitivity in three search tools for qualitative systematic reviews. BMC Health Services Research, 14(1), 579. https://doi.org/10.1186/s12913-014-0579-0

Morland, L. A., Mackintosh, M., Rosen, C. S., Willis, E., Resick, P., Chard, K., \& Frueh, B. C. (2015). Telemedicine versus in-person delivery of cognitive processing therapy for women with posttraumatic stress disorder: A randomized noninferiority trial. Depression and Anxiety, 32(11), 811-820. APA PsycInfo®. https://doi. org/10.1002/da.22397

Morrow, R. A., \& Brown, D. D. (1994). Critical theory and methodology (Vol. 3). Sage.

Murphy, D., \& Turgoose, D. (2019). Evaluating an Internet-based video cognitive processing therapy intervention for veterans with PTSD: A pilot study. Journal of Telemedicine and Telecare, https://doi. org/10.1177/1357633X19850393

Naeem, F., Phiri, P., Rathod, S., \& Ayub, M. (2019). Cultural adaptation of cognitive-behavioural therapy. BJPsych Advances, 25(6), 387-395. https://doi.org/10.1192/bja.2019.15

Neuhaus, C., \& Daniel, H. (2008). Data sources for performing citation analysis: an overview. Journal of Documentation, 64(2), 193-210. https://doi.org/10.1108/00220410810858010

Parker, E. (2019). New Zealand Mental Health Inequalities: Concentration Index and Ethnic Disparities. https://openrepository.aut. ac.nz/handle/10292/12547

Paterson, R., Durie, M., Disley, B., Rangihuna, D., Tiatia-Seath, J., \& Tualamali'i, J. (2018). He Ara Oranga: Report of the Government Inquiry into Mental Health and Addiction (p. 219) [Government Inquiry]. 978-0-9941245-3-1. https://mentalhealth.inquiry.govt. nz/inquiry-report/he-ara-oranga/

Petersen, D., Salazar, B., \& Kertz, S. J. (2020). Therapist and treatment-seeking students' perceptions of telemental health. Journal of Technology in Behavioral Science, 5(2), 113-120.

Possemato, K., Ouimette, P., \& Knowlton, P. (2011). A brief selfguided telehealth intervention for post-traumatic stress disorder in combat veterans: a pilot study. Journal of Telemedicine and Telecare, 17(5), 245-250.

Povey, J., Mills, P. P. J. R., Dingwall, K. M., Lowell, A., Singer, J., Rotumah, D., Bennett-Levy, J., \& Nagel, T. (2016). Acceptability of mental health apps for Aboriginal and Torres Strait Islander Australians: a qualitative study. Journal of Medical Internet Research, 18(3), e65.

Reay, R. E., Looi, J. C., \& Keightley, P. (2020). Telehealth mental health services during COVID-19: summary of evidence and clinical practice. Australasian Psychiatry, 28(5), 514-516. https://doi. org/10.1177/1039856220943032

Rozental, A., Boettcher, J., Andersson, G., Schmidt, B., \& Carlbring, P. (2015). Negative effects of internet interventions: A qualitative content analysis of patients' experiences with treatments delivered online. Cognitive Behaviour Therapy, 44(3), 223-236. Psychology and Behavioral Sciences Collection.

Smith, L. C. (2012). Decolonizing hybridity: Indigenous video, knowledge, and diffraction. cultural geographies, 19(3), 329-348.

Samuels, A. (1999). International telepsychiatry: A link between New Zealand and Australia. Australian \& New Zealand Journal of Psychiatry. https://doi.org/10.1080/0004867990063

Shultz, M. (2007). Comparing test searches in PubMed and Google Scholar. Journal of the Medical Library Association : JMLA, 95(4), 442-445. https://doi.org/10.3163/1536-5050.95.4.442

Simpson, S., Guerrini, L., \& Rochford, S. (2015). Telepsychology in a University Psychology Clinic Setting: A Pilot Project. Australian Psychologist, 50(4), 285-291. https://doi.org/10.1111/ap.12131 
Smoktunowicz, E., Barak, A., Andersson, G., Banos, R. M., Berger, T., Botella, C., Dear, B. F., Donker, T., Ebert, D. D., Hadjistavropoulos, H., Hodgins, D. C., Kaldo, V., Mohr, D. C., Nordgreen, T., Powers, M. B., Riper, H., Ritterband, L. M., Rozental, A., Schueller, S. M., \& Carlbring, P. (2020). Consensus statement on the problem of terminology in psychological interventions using the internet or digital components. Internet Interventions, 21, 100331. https://doi.org/10.1016/j.invent.2020.100331

Stafford-Smith, M., Griggs, D., Gaffney, O., Ullah, F., Reyers, B., Kanie, N., ... \& O'Connell, D. (2017). Integration: the key to implementing the Sustainable Development Goals. Sustainability science, 12(6), 911-919.

Stewart, R. W., Orengo-Aguayo, R. E., Cohen, J. A., Mannarino, A. P., \& de Arellano, M. A. (2017). A pilot study of traumafocused cognitive-behavioral therapy delivered via telehealth technology. Child Maltreatment, 22(4), 324-333. Education Source.

Te Pou. (2018). Effectiveness of e-mental health approaches - Rapid review (p. 56) [Rapid review]. https://www.tepou.co.nz/uploads/ files/resource-assets/E-therapy\%20report\%20FINAL\%20July\% 202018.pdf

Théberge-Lapointe, N., Marchand, A., Langlois, F., Gosselin, P., \& Watts, S. (2015). Efficacy of a cognitive-behavioural therapy administered by videoconference for generalized anxiety disorder. European Review of Applied Psychology, 65(1), 9-17.

Upton, D., \& Upton, P. (2015). Psychology of wounds and wound care in clinical practice. Springer.

Uscher-Pines, L., Sousa, J., Raja, P., Mehrotra, A., Barnett, M. L., \& Huskamp, H. A. (2020). Suddenly becoming a 'virtual doctor': Experiences of psychiatrists transitioning to telemedicine during the COVID-19 pandemic. Psychiatric Services, 71(11), 11431150. https://doi.org/10.1176/appi.ps.202000250

Wangelin, B. C., Szafranski, D. D., \& Gros, D. F. (2016). Telehealth technologies in evidence-based psychotherapy. In Computerassisted and web-based innovations in psychology, special education, and health (pp. 119-140). Elsevier.

Watson, H., Harrop, D., Walton, E., Young, A., \& Soltani, H. (2019). A systematic review of ethnic minority women's experiences of perinatal mental health conditions and services in Europe. PLoS One, 14(1), e0210587.

Watts, S., Marchand, A., Bouchard, S., Gosselin, P., Langlois, F., Belleville, G., \& Dugas, M. J. (2020). Telepsychotherapy for generalized anxiety disorder: Impact on the working alliance. Journal of Psychotherapy Integration, 30(2), 208.
Weinberg, H. (2020). Online group psychotherapy: Challenges and possibilities during COVID-19-A practice review. Group Dynamics: Theory, Research, and Practice, 24(3), 201.

Wells, S. Y., Morland, L. A., Wilhite, E. R., Grubbs, K. M., Rauch, S. A. M., Acierno, R., \& McLean, C. P. (2020). Delivering prolonged exposure therapy via videoconferencing during the COVID-19 pandemic: An overview of the research and special considerations for providers. Journal of Traumatic Stress, 33(4), 380-390. https:// doi.org/10.1002/jts.22573

Whittemore, R., \& Knafl, K. (2005). The integrative review: updated methodology. Journal of Advanced Nursing, 52(5), 546-553. https://doi.org/10.1111/j.1365-2648.2005.03621.x

Williams, A. D., Clark, T. C., \& Lewycka, S. (2018). The associations between cultural identity and mental health outcomes for indigenous Māori youth in New Zealand. Frontiers in Public Health, 6,319 .

Wind, T. R., Rijkeboer, M., Andersson, G., \& Riper, H. (2020). The COVID-19 pandemic: The 'black swan' for mental health care and a turning point for e-health. Internet Interventions, 20, 100317. https://doi.org/10.1016/j.invent.2020.100317

World Health Organisation. (1986). Ottawa Charter for Health Promotion. https://www.euro.who.int/en/publications/policy-documents/ ottawa-charter-for-health-promotion,-1986

World Health Organisation. (2010). Health systems financing: the path to universal coverage. Retrieved from:https://www.who.int/whr/ 2010/en/

Wosik, J., Fudim, M., Cameron, B., Gellad, Z. F., Cho, A., Phinney, D., Curtis, S., Roman, M., Poon, E. G., \& Ferranti, J. (2020). Telehealth Transformation: COVID-19 and the rise of Virtual Care. Journal of the American Medical Informatics Association, 27(6), 957-962.

Wozney, L., McGrath, P., Newton, A., Hartling, L., Curran, J., Huguet, A., \& Rao, S. (2017). RE-AIMing e-Mental Health: A Rapid Review of Current Research. https://www.mentalhealthcommissi on.ca/English/media/3931

Publisher's Note Springer Nature remains neutral with regard to jurisdictional claims in published maps and institutional affiliations. 